\title{
Mechanistic insights into filamentous phage integration in Vibrio cholerae
}

\author{
Bhabatosh Das * \\ Centre for Human Microbial Ecology, Translational Health Science and Technology Institute, Gurgaon, India
}

\section{Edited by:}

Jasna Rakonjac, Massey University,

New Zealand

Reviewed by:

Dahlene N. Fusco, Massachusetts

General Hospital, USA

Christophe Possoz, Centre National

de la Recherche Scientifique, France

\section{*Correspondence:}

Bhabatosh Das, Centre for Human

Microbial Ecology, Translational

Health Science and Technology

Institute, 496, Phase III, Udyog

Vihar, Gurgaon 122 016,

Haryana, India

e-mail: bhabatosh@thsti.res.in
Vibrio cholerae, the etiological agent of acute diarrhoeal disease cholera, harbors large numbers of lysogenic filamentous phages, contribute significantly to the host pathogenesis and provide fitness factors to the pathogen that help the bacterium to survive in natural environment. Most of the vibriophage genomes are not equipped with integrase and thus exploit two host-encoded tyrosine recombinases, $\mathrm{XerC}$ and $\mathrm{XerD}$, for lysogenic conversion. Integration is site-specific and it occurs at dimer resolution site (dif) of either one or both chromosomes of $V$. cholerae. Each dif sequence contains two recombinase-binding sequences flanking a central region. The integration follows a sequential strand exchanges between dif and attP sites within a DNA-protein complex consisting of one pair of each recombinase and two DNA fragments. During entire process of recombination, both the DNA components and recombinases of the synaptic complex keep transiently interconnected. Within the context of synaptic complex, both of the actuated enzymes mediate cleavage of phosphodiester bonds. First cleavage generates a phosphotyrosyl-linked recombinase-DNA complex at the recombinase binding sequence and free $5^{\prime}$-hydroxyl end at the first base of the central region. Following the cleavage, the exposed bases with 5'-hydroxyl ends of the central region of dif and attP sites melt from their complementary strands and react with the recombinase-DNA phosphotyrosyl linkage of their recombining partner. Subsequent ligation between dif and attP strands requires complementary base pair interactions at the site of phosphodiester bond formation. Integration mechanism is mostly influenced by the compatibility of dif and attP sequences. dif sites are highly conserved across bacterial phyla. Different phage genomes have different attP sequences; therefore they rely on different mechanisms for integration. Here, I review our current understanding of integration mechanisms used by the vibriophages.

Keywords: CTX $\Phi$, VGJ $\Phi$, TLC $\Phi$, XerC, XerD, dif, attP

\section{INTRODUCTION}

Bacterial pathogens evolve continuously to adapt to the changing environment by adopting multiple mobile genetic elements into their compact, modularly organized mosaic genomes to help combat the environmental factors that are detrimental to their subsistence (Frost et al., 2005; Mercier et al., 2008; Hassan et al., 2010). Vibrio cholerae, the noxious enteric pathogen with extraordinary fitness competence resides in multiple niches across continents. A variety of the vibrio strains have acquired multiple genetic traits in their genomes (Heidelberg et al., 2000; Sack et al., 2004; Chun et al., 2009), with the purpose of contributing to the toxin production (Waldor and Mekalanos, 1996), intestinal colonization (Rhine and Taylor, 1994), disease development (Herrington et al., 1988), antimicrobial resistance (Mazel and Davies, 1998; Beaber et al., 2004), cell division (Hassan et al., 2010), and survival in aquatic as well as gut environments (Davies et al., 2012). In V. cholerae, most of the horizontally acquired genetic traits integrate site-specifically within a short region of sequence identity shared by the host chromosome and the integrative mobile genetic elements (IMGEs), using self- or host-encoded tyrosine recombinases (Huber and Waldor, 2002; Rajanna et al., 2003; Hazen et al., 2010; Das et al., 2013; Banerjee et al., 2014). Integration might be reversible or irreversible, depending upon the structures of pre- and post-integrative attachment sequences (Das et al., 2011a, 2013, 2014). Tyrosine recombinases can bind double stranded as well as folded single stranded DNA of the acquired exogenous genetic elements (Val et al., 2005; Mazel, 2006; Das et al., 2011b). Different acquired genetic traits reported in $V$. cholerae are heterogeneous, and they recognize different receptors for infection (Herrington et al., 1988; Campos et al., 2010; Das et al., 2013), follow different mechanisms to deliver DNA into the host cytoplasm (Heilpern and Waldor, 2000), have a wide range of genomic content (Heidelberg et al., 2000; Faruque and Mekalanos, 2003), and most importantly rely on catalytic activities of different recombinases for chromosomal integration (Rajanna et al., 2003; Das et al., 2010, 2011b; Midonet et al., in press). In a broader sense, these IMGEs can be classified into two different groups, depending upon the presence and absence of recombinases, the enzymes essential for their integration to the host genome. IMGEs, which encode and rely on 
Table 1 | Key components of the Xer recombination system in $V$. cholerae.

\begin{tabular}{lcccrr}
\hline Gene & Gene ID in N16961 genome & Gene length (bp) & Protein & Protein length (aa) & Mol. weight (kD) \\
\hline xerC & $V C 0128$ & 936 & XerC & 311 & 35.55 \\
xerD & $V C 2419$ & 909 & XerD & 302 & 34.56 \\
ftsK & $V C 1903$ & 2883 & FtsK & 960 & 105.89 \\
\hline
\end{tabular}

their own recombinases for integration, are generally large in size and integrates specifically in front of tRNA or tmRNA operon (Karaolis et al., 1999; Heidelberg et al., 2000). On the other hand, IMGEs exploit host-encoded tyrosine recombinases (IMEX), are small in size and integrate at the dimer resolution sites (dif) of host chromosomes (Das et al., 2013, 2014).

The best-characterized IMEX, which exploits the host encoded tyrosine recombinases for its lysogenic conversion is CTX $\Phi$, a temperate filamentous bacteriophage that encodes the cholera toxin in $V$. cholerae (Waldor and Mekalanos, 1996). The CТХФ recognizes the toxin co-regulated pilus (TCP), a type IV pilus encoded by the vibrio pathogenic island-I (VPI-1), and subsequently introduces its $\sim 7.0 \times 10^{3}$ nucleotides long circular single stranded genomic DNA ( $+\mathrm{ssDNA})$ into the $V$. cholerae cytoplasm. Once in the bacterial cytoplasm, ssDNA may convert to dsDNA, and start the rolling circle replication, or it can be recognized by the chromosomally-encoded tyrosine recombinases, that enable CTX $\Phi$ integration into the dif site of host chromosome. Tandemly integrated CTX $\Phi$ can also initiate the rolling circle replication from the chromosome and produce extrachromosomal ssDNA genome that may contribute in virion production (Davis and Waldor, 2000). Compared to other episomally replicative filamentous phages, the number of phage particles produced by the CTX-prophage is very low, even upon induction, resulting in one phage particle produced per 10-100 host cells (Davis et al., 2002). The other two wellcharacterized IMEXs that use the same recombinases for integration at the dif1 site of $V$. cholerae are VGJ $\Phi$ and TLC $\Phi$ (Campos et al., 2003b; Hassan et al., 2010; Das et al., 2011b). Presence of single or multiple copies of VGJ $\Phi$ and TLC $\Phi$ were reported in toxigenic $V$. cholerae isolates. Unlike $C T X \Phi$, the virion production of VGJ $\Phi$, and TLC $\Phi$ mainly relies on the episomal replication. Although the genomes of the two latter elements do not encode any toxins, they are nevertheless implicated in the host fitness and play important role in acquisition of the cholera-toxin-encoding genes and CTX $\Phi$ dissemination (Hassan et al., 2010; Das et al., 2011b; Midonet et al., in press).

In this review, I will update the mechanistic insights into filamentous vibriophage integration and cooperative interactions amongst IMGEs, which support the emergence of new pathogenic strains by contributing efficient acquisition and rapid dissemination of the cholera toxin genes in closely or distantly related bacterial strains.

\section{XER RECOMBINATION SYSTEM IN VIBRIO CHOLERAE}

The native function of Xer recombination system is to ensure the stable maintenance of monomeric circular replicon (Blakely et al., 1993). In $V$. cholerae, the principal components of the
Xer recombination system are (i) two recombinases, XerC and XerD; (ii) a DNA motor protein, FtsK; (iii) a 28-bp DNA sequence, dif (Val et al., 2008) (Table 1). The Xer proteins are members of the tyrosine recombinase family (Esposito and Scocca, 1997). Members of this family share two conserved motifs, containing four highly conserved residues (R1-H-R2$\mathrm{Y})$, a catalytic tyrosine, which acts as the nucleophile during cleavage of phosphodiester bond, two arginines and a histidine, which have been implicated both the cleavage and rejoining of DNA strands (Esposito and Scocca, 1997). The two positively charged arginines are thought to stabilize the pentavalent phosphate transition state, whereas the histidine may act as a general base catalyst (Esposito and Scocca, 1997; Sherratt and Wigley, 1998).

During chromosome dimer resolution at the end of DNA replication, tetrameric XerC and XerD recombinase complex catalyzes successive strand exchanges between the two dif sites of a dimeric chromosome. The resolution is completed by a two-step transesterification reaction in which the $\mathrm{OH}$ - group of catalytic tyrosine residue of each recombinase is directly involved in the phosphodiester bond formation (Figure 1). In the first step of dimer resolution reaction, cleavage of a phosphodiester bond in DNA is introduced by the active enzyme, generating a covalently linked enzyme-DNA complex at the recombinase binding site and a free $5^{\prime}$-hydroxyl group at the end of the central region. Following the cleavage, the exposed sequence at the $5^{\prime}$-hydroxyl ends of the central region melt from their complementary strands and react with their recombinase-DNA phosphotyrosyl linked recombining partner. Subsequent joining between dif and attP strands requires complementary base pair interactions at the site of the phosphodiester bond formation (MacDonald et al., 2006; Das et al., 2010).

FtsK, a bifunctional protein essential for cell division and chromosome partitioning, induces the XerD function during chromosome dimer resolution (Recchia et al., 1999; Aussel et al., 2002; Demarre et al., 2013). The N-terminal part of FtsK forms a transmembrane domain and is directly linked to the forming septum. The cytoplasmic C-terminal domain is involved in the inter- and intracellular DNA transfer and activation of XerD recombinases (Bigot et al., 2006). Recent reports demonstrated that several IMGEs exploit the conserved Xer recombination system of bacteria to mediate their integration in the dimer resolution site of host chromosomes (Das et al., 2013). The integration mechanisms of such elements were studied in details in $V$. cholerae and revealed that the IMEXs present in the dif region of $V$. cholerae make use of three distinct integration mechanisms for lysogenic conversion (Das et al., 2010, 2011a,b, 2013; Midonet et al., in press). 


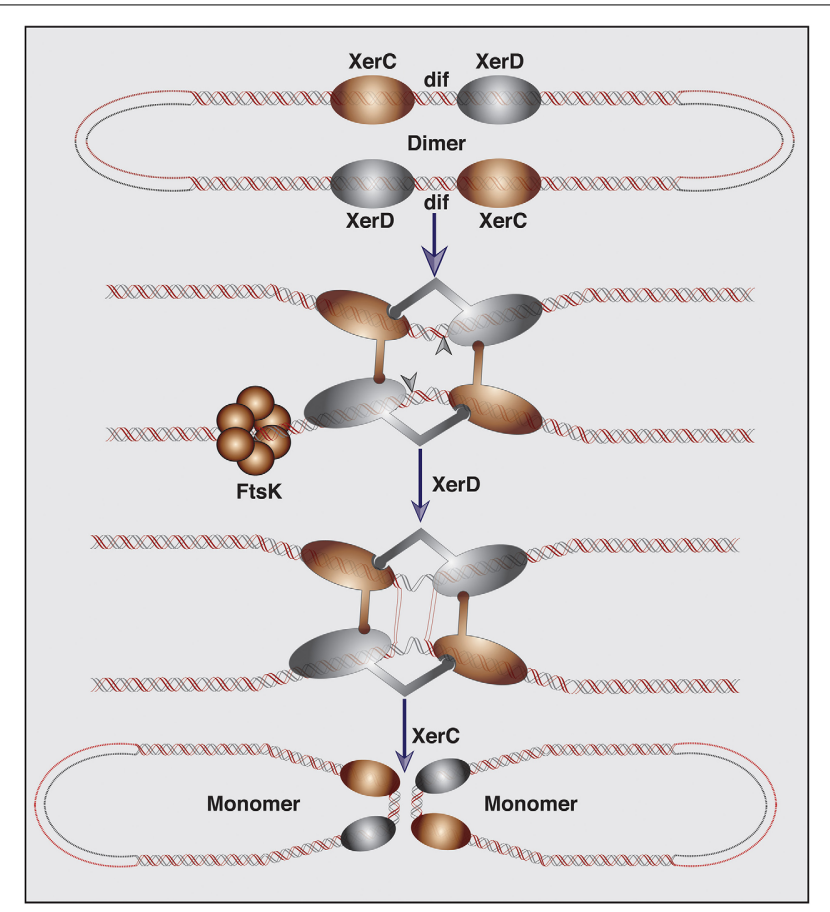

FIGURE 1 | Schematic representation of the XerC-XerD mediated chromosome dimer resolution. Chromosome dimers are resolved by the XerC-XerD-mediated recombination at the dif site. DNA motor protein FtsK brings two dif sites together and induces the XerD catalytic activity required for the first pair of strand exchanges. Followed isomerization, XerC mediates the second pair of strand exchanges, and completes the recombination reaction.

\section{LYSOGENIC FILAMENTOUS VIBRIOPHAGES EXPLOIT XER RECOMBINASES}

Most of the characterized filamentous vibriophages are lysogenic and integrate at the dif1 and/or dif2 sites of $V$. cholerae. All reported filamentous vibriophages are equipped with an autonomously replicating genetic module, with or without toxinencoding genes (Figure 2). Some of the filamentous vibriophages are directly responsible for $V$. cholerae pathogenesis, others contribute to toxin acquisition and host fitness (Waldor and Mekalanos, 1996; Hassan et al., 2010). Their genomes may or may not be equipped with genes required for virion production and can exploit the virion structural and assembly genes of other filamentous phages for genome packaging (Hassan et al., 2010). Although most of the filamentous vibriophages use host-encoded Xer machinery for their integration, their attachment sites (attP), and their genomic organization are fairly distinct (Figure 2). The best-studied CTХ $\Phi$ genome is organized into two structurally and functionally distinct modules called repeat sequence 2 (RS2) and core (Figure 2). The RS2 module carries genetic traits essential for CTX $\Phi$ replication, maintenance of the ssDNA genome, and transcriptional regulation from phage originated $\mathrm{P}_{\mathrm{RSTA}}$ promoter (Waldor et al., 1997). The replicating genome of CTX $\Phi$ is detrimental to $V$. cholerae growth, but is tolerated upon integration into the dif sites of either one or both chromosomes (Das et al., 2010; Faruque and Mekalanos, 2012). Compared to toxigenic strains, that contain a single or multiple integrated CTX $\Phi$ genomes, $V$. cholerae cells carrying a replicative form of CTX $\Phi$ grow slowly and rapidly lose the replicative phage both under the standard laboratory growth conditions and in rabbit gastrointestinal tract animal model (Faruque et al., 2001).

VGJ $\Phi$, a filamentous (+)ssDNA lysogenic bacteriophage, infects both clinical and environmental isolates of cholera pathogen and exploits the host-encoded Xer recombinases to integrate at the dif1 site in the large chromosome of $V$. cholerae (Campos et al., 2003b, 2010; Das et al., 2011b). In contrast to complex attachment site of СТX $\Phi$ that contains two dif-like attP sites in an inverted orientation, the 7.5-kb genome of VGJ $\Phi$ harbors a single dif-like 29-bp attachment sequence, called attP $P^{V G J}$ and integrates specifically into the dif 1 site.

TLC $\Phi$, a satellite temperate phage with an autonomous replication module but lacking the morphogenesis and structural virion-encoding genes, is often present in the dif1 region of toxigenic $V$. cholerae isolates (Rubin et al., 1998). Recent reports demonstrated that, like CTX $\Phi$, and VGJ $\Phi$, TLC $\Phi$ also relies on the host-encoded Xer machinery for establishing lysogeny (Hassan et al., 2010; Midonet et al., in press). Interestingly, although both the XerC and XerD recombinases are essential for integration of IMEXs, none of the reported IMEXs rely on FtsK for recombination.

Different vibriophages have different attP structures and therefore rely on to a certain extent of different mechanisms of interaction with the Xer recombinases and integration (Das et al., 2013).

\section{MECHANISTIC INSIGHTS INTO LYSOGENIC CONVERSION OF VIBRIOPHAGES}

Most lysogenic filamentous vibriophages are IMEXs that depend on the host-encoded Xer recombinases for their lysogeny. The attachment site in the phage genome mimics the native chromosomal Xer binding sites and exploits Xer recombinases to catalyze site-specific recombination and enable phage integration. Based on the attP structure and integration mechanisms, vibriophages can be categorized into three classes (Table 2). Although the constituents of the integration reaction are very similar, integration is achieved by three distinct mechanisms, where catalytic activity of recombinases, order of strand exchanges and the resolution of reaction intermediates into end products are different (Figures 3-5). In this review, I will concentrate on CTX $\Phi$, VGJ $\Phi$, and TLC $\Phi$ lysogenic conversions to describe the most updated integration mechanisms of these phages studied in V. cholerae and related bacterial cells.

\section{MECHANISTIC INSIGHTS INTO CTX $\Phi$ INTEGRATION}

CTX-prophages are ubiquitously present in the chromosomes of all epidemics V. cholerae isolates (Mekalanos et al., 1983; Mekalanos, 1985; Vanden Broeck et al., 2007). Phage to prophage conversion is rapid and efficient process achieved through sitespecific recombination between attP(+) of CTX $\Phi$ and dif sites of $V$. cholerae (Val et al., 2005) that results in a stable integration. The functional attP(+) structure is formed by intra-strand base pairing interactions between two inversely oriented attP 


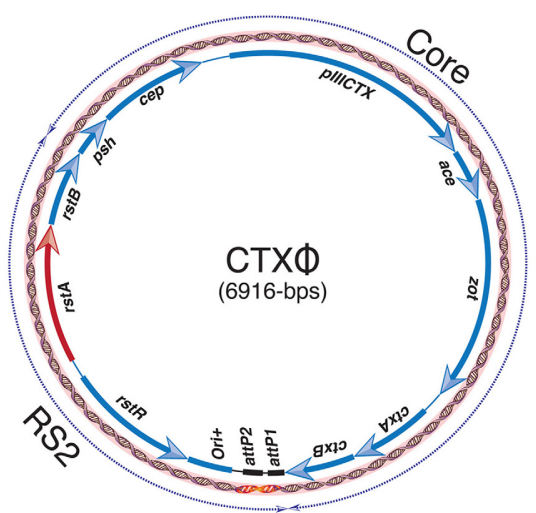

FIGURE 2 | Genomic organization of $C T X \Phi$, VGJ $\Phi$, and TLC $\Phi$ phages. A red arrow depicts gene essential for replication in each genome. A solid black line denotes attachment sequence, recognized by the Xer recombinases. Replicative form (RF) of each phage

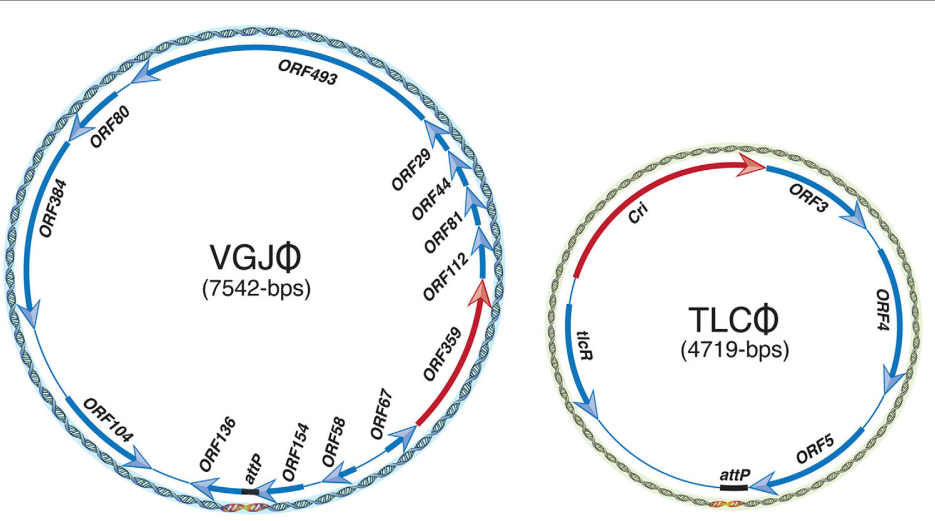

genome is presented as dsDNA. Arrows indicate the direction of transcription of each ORF. A thin solid line illustrates intergenic regions. Dashed arrows above the and RS2 modules.

Table 2 | Different components and strategies used by three different filamentous Vibriophages for their integration at dif sites of $V$. cholerae chromosome.

\begin{tabular}{|c|c|c|c|}
\hline Class & СТX $\Phi$ & VGJ $\Phi$ & $\operatorname{TLC} \Phi$ \\
\hline Phage attachment site & $\operatorname{attp}(+)(\sim 150$ nt) & $a_{t t p}^{V G J}(29-b p)$ & $a_{t t p}^{T L C}(28-b p)$ \\
\hline Essential components for integration & XerC, XerD, attp(+), dif1/dif2 & XerC, XerD, attp VGJ, dif1 & XerC, XerD, att ${ }^{T L C}$, dif1 \\
\hline First pair of strand exchanges is mediated by & XerC & XerC & XerD \\
\hline Integration is completed by & Host DNA replication & Host DNA replication & XerC \\
\hline Excision initiated by & - & XerC & XerD \\
\hline Excision completed by & - & Host DNA replication & XerC \\
\hline Prophage to phage production depends on & Rolling circle replication & Excision & Excision \\
\hline Related members & RS1, Ypf $\Phi$, CUS-1 $\Phi$ & VEJ $\Phi$, VSKK, VSK, fs2, Vf12, VfO4K68 & $\Phi\llcorner f, X f \Phi f 1, C f 1 c, G G l$ \\
\hline
\end{tabular}

sequences located between the $\operatorname{ctx} B$ and $r s t R$ genes in the circular CTX $\Phi$ genome (Figure 2). In a replicating form (RF) of the genome, attP1 consists of XerC and XerD binding sites separated by a $12 \mathrm{bp}$ central region. The attP2 is consists of 11-bp XerC and XerD binding sites and a 5-7 bp central region. Neither attP1 nor $a t t P 2$ are suitable for Xer-mediated integration due to extended central region or presence of incompatible bases in the central region, respectively (Das et al., 2010). The specificity and compatibility of $\mathrm{CTX} \Phi$ integration is solely determined by the homology of bases next to the XerC-binding site between the overlapping (or corresponding) regions of attP and dif sequence (Das et al., 2010). A 90-bp DNA fragment separates the attP1 and attP2 of СТХ $\Phi$. The СТХ $\Phi$ integration sites in $V$. cholerae chromosomes (dif1 and dif2) each consist of 28 bp DNA sequence. In dif1, a 6 bp central region, at the border of which the strand exchange occurs, separates the $11 \mathrm{bp}$ binding sites of XerC and XerD. The chromosomally-encoded Xer recombinases can recognize both the double stranded chromosomal dif site and folded single stranded $a t t P(+)$ of CTX $\Phi$ and catalyze recombination to facilitate the phage ssDNA integration.
The CTX $\Phi$ ssDNA integration takes place within a DNAprotein complex, where DNA strands are cleaved and rejoined sequentially by XerC recombinase through transient nucleoprotein covalent intermediates. The catalytic tyrosine residue of Xer recombinases functions as a nucleophile and mediates the cleavage of a phosphodiester bond between the last base of the recombinase-binding site and the first base of central region. The key components within the nucleoprotein complex are XerC, $\mathrm{XerD}, \operatorname{attP}(+)$, and dif sequences. Although the presence of both recombinases are essential for recombination, successful integration of the CTX $\Phi$ ssDNA needs only the catalytic activity of XerC (Val et al., 2005). At the onset of recombination reaction, XerC introduces a cut of the phosphodiester bond between the last base of XerC binding site and the first base of the central region, of dif1/dif2, and attP(+) (Figure 3). On one side, a XerC-DNA complex is formed through a covalent phosphotyrosyl bond. On the other side, the DNA strand with a free $5^{\prime}-\mathrm{OH}$ end melts away from the central region of the reaction complex, migrates to the central region of the partner strand and forms complementary base pairing interactions to stabilize the exchanged 


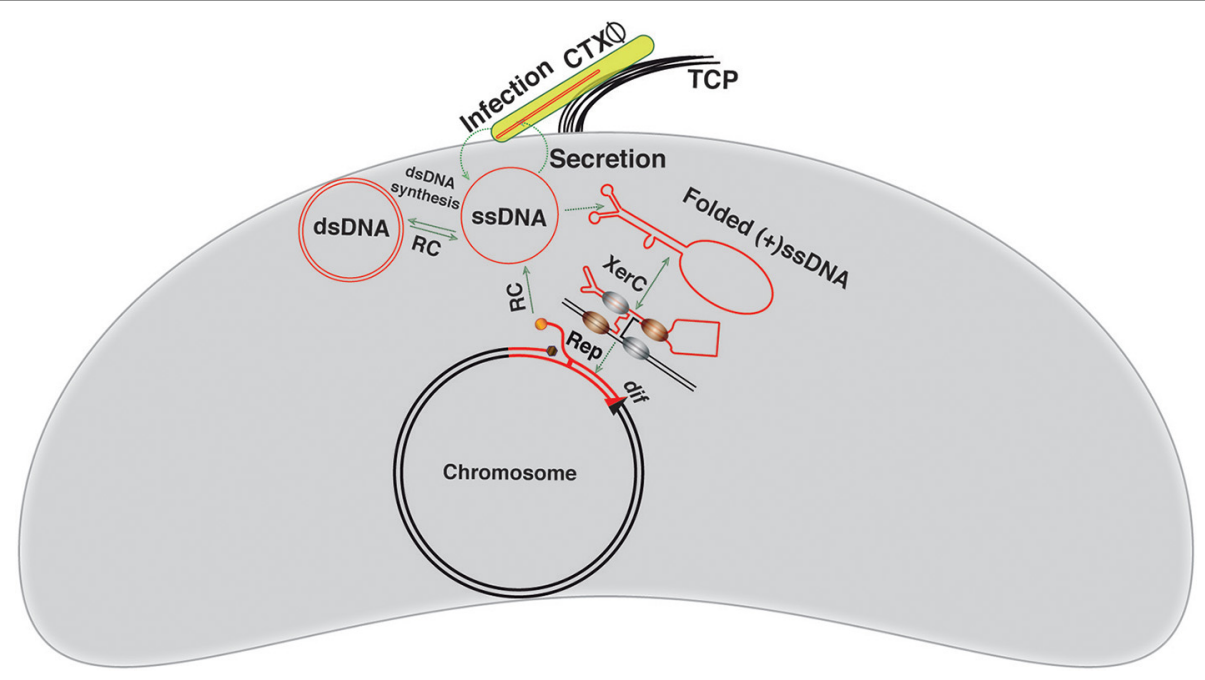

FIGURE 3 | Visual depiction of the CTX $\Phi$ integration and replication. The $\mathrm{CTX} \Phi$ virion recognizes the cognate $V$. cholerae cell surface receptor TCP and delivers its ssDNA genome into the $V$. cholerae cytoplasm. The ssDNA genome of $\mathrm{CTX} \Phi$ may be converted into dsDNA or directly integrated into a chromosomal dif site using the host-encoded XerC-XerD recombinases. XerC mediates first pair of strand exchanges. The host DNA replication, possibly, resolves the resulting Holiday junction. The red line depicts the phage genome, while a black line shows bacterial genome. Newly generated dif site is represented by a triangle.

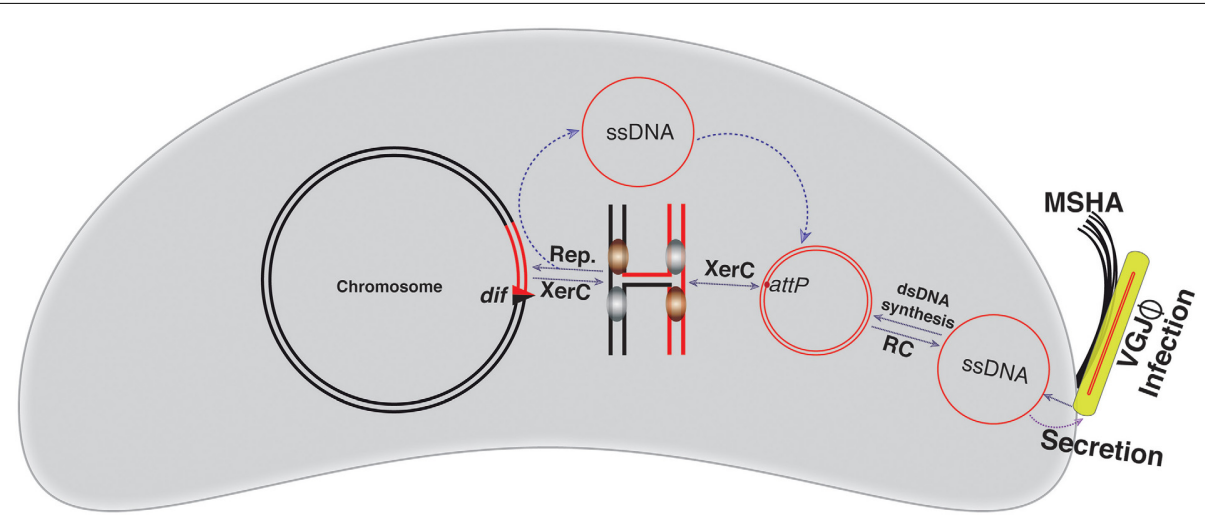

FIGURE 4 | Schematic representation of the VGJ $\Phi$ integration and replication. VGJ $\Phi$ uses mannose-sensitive hemagglutinin $\mathrm{A}$ (MSHA) to as a primary host receptor and delivers its SsDNA genome into the host cytoplasm. The ssDNA genome of VGJ $\Phi$ is first converted into dsDNA and for this it contains a dif like DNA sequence, attPVGJ. XerC mediates the first pair of strand exchanges and the host DNA replication resolves the resulting Holiday junction. The VGJ $\Phi$ integration is reversible. Excision follows the same sequence of strand exchanges as described for integration. The red line depicts the phage genome while a black line depicts bacterial genome. Newly generated dif site is represented by a triangle. strands and form of a phosphodiester bond between the deoxyriboses of two adjacent nucleotides. This step is the determining factor for phage integration specificity (Das et al., 2010). The resulting strand exchanges generate a Holiday Junction $(\mathrm{HJ})$ intermediate. Host DNA replication, possibly, resolves the resulting, transient $\mathrm{HJ}$ intermediate and accomplishes the CTX $\Phi$ ssDNA integration into the $V$. cholerae chromosome (Figure 3 ). The absence of any homology between central region bases at the XerD-side of attP(+) and dif1/dif2 impedes any XerD mediated strand exchange. A recent report revealed that the host-encoded DNA repair protein, endonuclease III, a product of $n$th gene with $\mathrm{N}$-glycosylase and AP-lyase functions, facilitates the CTX $\Phi$ genome integration, possibly by stabilizing the transient $\mathrm{HJ}$ intermediate (Bischerour et al., 2012). Each individual step in the СТХ $\Phi$ genome integration pathway is in principle reversible, but the prophage excision has never been detected under the standard laboratory conditions. This is due to the loss of a functional folded $\operatorname{att} P(+)$ structure in the double-stranded form of prophage (Val et al., 2005), in which the intra- strand base pairing between attP1 and $a t t P 2$ is excluded. In the prophage genome, both attP sequences retained the XerC and XerD binding sites, but the central region of both sites, where the strand exchange occurs during integration, are incompatible with the Xer reaction and make the CTX $\Phi$ integration irreversible (Das et al., 2010). 


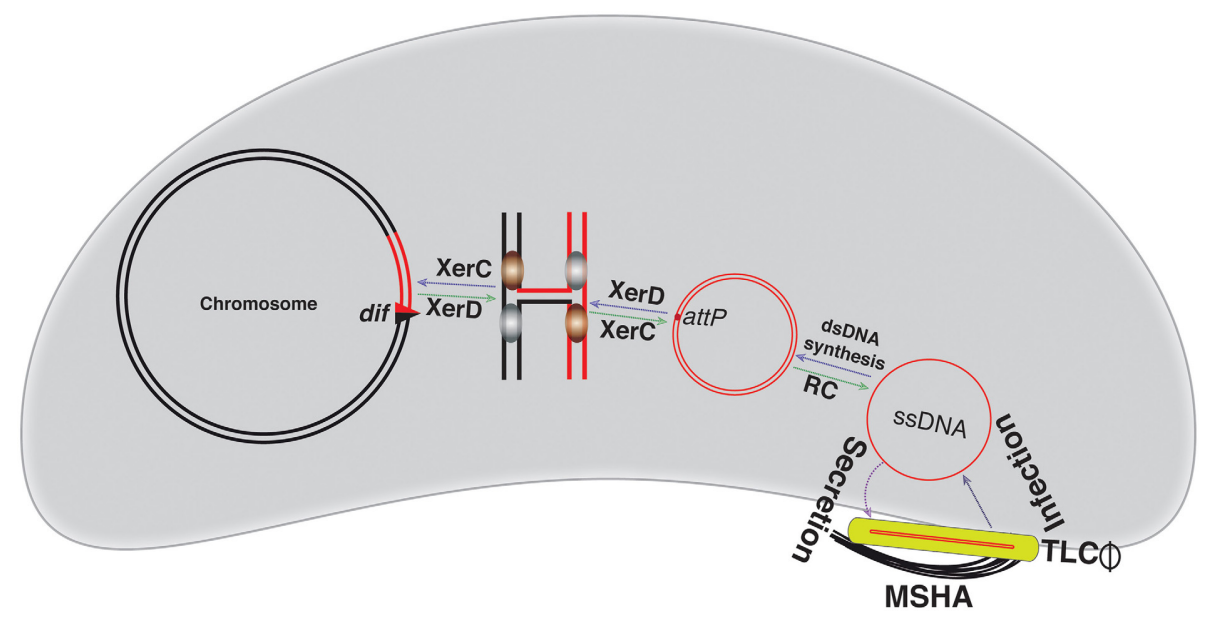

FIGURE 5 | Illustration of the TLC $\Phi$ integration and replication. The TLC $\Phi$ satellite prophage uses morphogenesis proteins of other filamentous phages, like fs 2 for assembly. It uses the mannose-sensitive hemagglutinin A (MSHA) as the primary host receptor and delivers its ssDNA genome into host cytoplasm. The ssDNA genome of the TLC $\Phi$ is first converted into dsDNA. The attP $P^{T L C}$ in the dsDNA replicative genome of TLC $\Phi$ is recognized by the XerC-XerD recombinases. XerD mediates the first pair of strand exchanges and generates a Holiday junction. After isomerization, XerC mediates second pair of strand exchanges and enables the TLC $\Phi$ integration. TLC $\Phi$ integration, which is reversible. Excision follows the same sequence of strand exchanges as described for integration. The blue and green arrows indicate integration and excision pathways, respectively. The red line depicts phage genome, while a black line represents bacterial genome. Newly generated dif site is symbolized by a triangle.

\section{MECHANISTIC INSIGHTS INTO VGJ $\Phi$ INTEGRATION}

VGJ $\Phi$, a filamentous (+)ssDNA lysogenic bacteriophage, infects both clinical and environmental isolates of cholera pathogen and exploits the host-encoded Xer recombinases to integrate at the dif1site in the large chromosome of cholera pathogen (Campos et al., 2003b; Das et al., 2011b). In contrast to CTX $\Phi$, the $7.5 \mathrm{~kb}$ genome of VGJ $\Phi$ harbors a single dif like 29-bp DNA sequence, called $a t t P^{V G J}$ (Figure 2). Presence of the single functional dif like site allows integration of VGJ $\Phi$ genome into the $V$. cholerae chromosome as dsDNA. Like CTX $\Phi$, integration of VGJ $\Phi$ also relies on the catalytic activity of XerC (Figure 4). Due to an absence of the sequence homology in the central region adjacent to the XerD-binding site between the attP $P^{V G}$ and dif1 site of $V$. cholerae, XerD catalytic activities are not been used either to generate $\mathrm{HJ}$ or to resolve the $\mathrm{HJ}$ during integration. The XerC generated HJ junction is resolved either by the host DNA replication or other DNA repair proteins and assist VGJ $\Phi$ integration (Figure 4). After integration, two compatible Xer recombination sites ( $a t t L$ and $a t t R$ ) flank the VGJ-prophage. The 7.5-kb distance between the two Xer binding sites does not impede assembly of the recombination complex and excision of the VGJ $\Phi$ prophage. Like integration, excision of VGJ $\Phi$ also depends on the XerC-mediated strand exchanges (Figure 4). In vitro recombination reactions between synthetic attP $P^{V G J}$ and dif1 substrates confirmed that only XerC and XerD recombinases are sufficient for DNA rearrangement (Das et al., 2011b).

\section{MECHANISTIC INSIGHTS INTO TLC $\Phi$ INTEGRATION}

TLC $\Phi$, a satellite temperate phage with an autonomous replication module, is mostly present in the dif1 region of toxigenic $V$. cholerae isolates. Recent reports demonstrated that like CTX $\Phi$, and VGJ $\Phi$, TLC $\Phi$ also relies on host encoded Xer machinery for its lysogeny (Hassan et al., 2010; Midonet et al., in press). In sharp contrast to the first two elements, the XerD binding site of TLC $\Phi$ is degenerated (Das et al., 2013; Midonet et al., in press). The XerC binding site and central region of attP $P^{T L C}$ are almost identical to dif1 of $V$. cholerae, but XerD binding sequence has very little similarity (Midonet et al., in press). Despite the absence of bona fide XerD binding site in attP $P^{T L C}$, TLC $\Phi$ integration is strictly related to XerD catalytic activity (Midonet et al., in press). The integration mechanism of TLC $\Phi$ is unique compared to the integration strategy adopted by CTX $\Phi$, and VGJ $\Phi$ (Table 2 ).

Like CTX $\Phi$ and VGJ $\Phi$, TLC $\Phi$ integration also needs both XerC and XerD recombinases. During TLC $\Phi$ integration, XerC and XerD form a hetero-tetrameric complex with dif1 and $a t t P^{T L C}$, within which XerD exchanges first pair of DNA strands and form the HJ. The resulting HJ proceed to DNA isomerization and is subsequently resolved by the XerC-mediated second strand exchanges (Figure 5). Tyrosine recombinases-mediated reactions are reversible, and therefore the TLC $\Phi$ integration is also reversible. Like integration excision is also depends on XerD catalytic activity (Figure 5). The action of both recombinases and the sequence of strand-exchanges stages in both integration and excision are very similar to chromosome dimer resolution, however the TLC $\Phi$ integration occurs without direct participation of DNA motor protein FtsK. Since the recombination reaction between dif1 and att $P^{T L C}$ is not reconstituted in defined in vitro reactions, it is yet not clear whether only the $28-\mathrm{bp}$ att $P^{T L C}$ is sufficient for Xer reaction or whether the TLC $\Phi$ needs extended att $P^{T L C}$ region and support from additional host proteins for efficient integration. Similarly, the mechanistic insights into TLC $\Phi$ excision in defined reaction conditions are yet to be explored.

\section{COOPERATIVE INTERACTIONS AMONG FILAMENTOUS VIBRIOPHAGES}

Metagenomic studies revealed several IMEXs integrated at one or both chromosomes of cholera pathogenic strains (Chun et al., 


\section{Box 1 | Outstanding questions.}

* How is the CTX $\Phi$ integration efficiency modulated? Does TLC $\Phi$ modulate efficiency of CTX $\Phi$ integration into the $V$. cholerae chromosomes?

* How is the CTX $\Phi$ virion produced from toxigenic $V$. cholerae cells, in the case when it harbors only a single copy of the prophage in either chromosome?

* Does the TLC $\Phi$ contribute to the CTX $\Phi$ rolling circle replication?

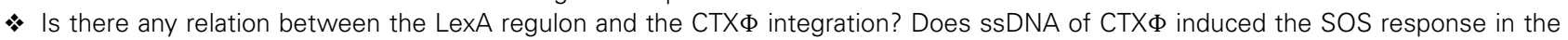
host cell?

* How does the TLC $\Phi$ genome integrate in the chromosome of classical $V$. cholerae strains, where both chromosomes contained an attPTLC incompatible dif2 sequence?

* Are there any accessory proteins other than Xer recombinases that may help the TLC $\Phi$ genome integration into the $V$. cholerae chromosome?

- What are the host factors implicated in the CTX $\Phi$, VGJ $\Phi$, and TLC $\Phi$ replication?

2009). The co-occurrence of multiple IMEXs in the genome of cholera pathogens is not simply a coincidence. Recent reports demonstrated that there are remarkable cooperative interactions between closely or distantly located IMEXs in cholera pathogen (Taylor et al., 1986; Hassan et al., 2010; Das et al., 2011b). Interactions between IMEXs happen at different levels, including the host recognition, receptor binding and DNA entry into the cytoplasm, chromosomal integration, and virion production (Waldor and Mekalanos, 1996; Hassan et al., 2010). The best example of this interaction is acquisition of cholera toxin. The first step of СТХ $\Phi$ infection in $V$. cholerae is to recognize its host receptor. TCP, the cell surface receptor of $V$. cholerae for CTX $\Phi$, is encoded by VPI-1, a $\sim 41-\mathrm{kb}$ mobile genomic island. The same genomic island also helps cholera toxin production by providing transcriptional inducer ToxT, which specifically binds to the "TATTA" repeat, invariably present upstream of the $c t x A B$ operon.

Cooperative interactions between VGJ $\Phi$ and CTX $\Phi$ have been reported very recently (Campos et al., 2003a; Das et al., 2011b). СТX $\Phi$ integration is irreversible and phage production depends on the presence of tandem copies of prophages or related RS1 elements (Waldor et al., 1997; Val et al., 2005). A recent report demonstrated that VGJ $\Phi$ could help CTX $\Phi$ excision and its dissemination to the $V$. cholerae strains devoid of TCP island. When the CTX $\Phi$ prophage is inserted between the attachment site of VGJ $\Phi$ and the dif1 of $V$. cholerae, a hybrid VGJФ-CTX $\Phi$ is often detected (Das et al., 2011b). The hybrid phage genome is packed within the VGJ $\Phi$ coat proteins and infects $V$. cholerae cells expressing mannose-sensitive hemagglutinin (MSHA) pilus, a type IV pilus present on the cell surface whose structural pilin subunit is encoded by the $m s h A$ gene. MSHA pilus is ubiquitous and constitutively expressed in all $V$. cholerae serotypes and thus, host range of the hybrid phage containing the СТX $\Phi$ genome encapsulated in the VGJ $\Phi$-encoded virion is not restricted to the cells that expressed TCP (Fullner and Mekalanos, 1999). Similarly, TLC $\Phi$ also helps the CTX $\Phi$ excision if both elements are present in tandem in the $V$. cholerae chromosome (Midonet et al., in press). Since the TLC prophage is present in most toxigenic strains where a single or multiple copies of the CTX $\Phi$ genome are integrated at the chromosomal dif1 site, the TLC $\Phi$ is more significant for the CTX $\Phi$ excision than is the VGJ $\Phi$-prophage. Other than TLC $\Phi$ and VGJ $\Phi$ elements, several other IMGEs could help CTX $\Phi$ replication as well as its interaction with the $V$. cholerae in natural environment by providing cell surface receptor. They can also help increase the amount of cholera toxin production by up-regulating $\operatorname{ct} A B$ operon, protein required for the hybrid virion production and dissemination in the environment.

\section{CONCLUSIONS}

In this review, several fundamental questions related to IMEXs integration have been addressed, such as contribution of these elements to continuous evolution of the cholera pathogenic strains. Although hypothesis for the adaptive advantage of lysogenic conversion of filamentous vibriophages was proposed, many important questions are still open and need to be addressed in order to improve understanding of the $V$. cholerae biology and cholera management (Box 1). Pangenomic studies revealed that IMEXs are not limited only to clinical isolates but are also widely distributed in the environmental $V$. cholerae isolates that are closely or distantly related to pathogenic strains. Much emphasis has been put on the biology of those IMEXs that are mostly present in the clinical isolates. Our knowledge on IMEXs present in the environmental isolates is limited. It is widely accepted that the environmental $V$. cholerae strains are ubiquitously distributed in aquatic environment and could serve as a reservoir of toxinencoding genes as well as other fitness factors for clinical strains. Comprehensive studies of IMEXs present both in clinical and environmental isolates of $V$. cholerae and related pathogenic strains are required in order to understand the microevolution of the species pertinent to epidemiology. On the other hand, better mechanistic and structural knowledge of IMEXs will help to develop therapeutic agents and limit the emergence of the new cholera pathogenic strains and other strains that may pose a serious threat to human beings as well as animals.

\section{ACKNOWLEDGMENTS}

I thank Dr. G.B. Nair and other members of CHME-THSTI for invaluable suggestions and help during the preparation of this review. The work is supported by the Department of Science and Technology (Grant No. SB/FT/LS-309/2012), Government of India (GOI) and the Department of Biotechnology (Grant No. BT/MB/THSTI/HMC-SFC/2011), GOI. 


\section{REFERENCES}

Aussel, L., Barre, F. X., Aroyo, M., Stasiak, A., Stasiak, A. Z., and Sherratt, D. (2002). FtsK Is a DNA motor protein that activates chromosome dimer resolution by switching the catalytic state of the XerC and XerD recombinases. Cell 108, 195-205. doi: 10.1016/S0092-8674(02)00624-4

Banerjee, R., Das, B., Balakrish Nair, G., and Basak, S. (2014). Dynamics in genome evolution of Vibrio cholerae. Infect. Genet. Evol. 23C, 32-41. doi: 10.1016/j.meegid.2014.01.006

Beaber, J. W., Hochhut, B., and Waldor, M. K. (2004). SOS response promotes horizontal dissemination of antibiotic resistance genes. Nature 427, 72-74. doi: 10.1038/nature02241

Bigot, S., Saleh, O. A., Cornet, F., Allemand, J. F., and Barre, F. X. (2006). Oriented loading of FtsK on KOPS. Nat. Struct. Mol. Biol. 13, 1026-1028. doi: $10.1038 / \mathrm{nsmb} 1159$

Bischerour, J., Spangenberg, C., and Barre, F. X. (2012). Holliday junction affinity of the base excision repair factor Endo III contributes to cholera toxin phage integration. EMBO J. 31, 3757-3767. doi: 10.1038/emboj.2012.219

Blakely, G., May, G., McCulloch, R., Arciszewska, L. K., Burke, M., Lovett, S. T., et al. (1993). Two related recombinases are required for site-specific recombination at dif and cer in E. coli K12. Cell 75, 351-361. doi: 10.1016/0092-8674(93) 80076-Q

Campos, J., Martinez, E., Izquierdo, Y., and Fando, R. (2010). VEJ\{phi\}, a novel filamentous phage of Vibrio cholerae able to transduce the cholera toxin genes. Microbiology 156, 108-115. doi: 10.1099/mic.0.032235-0

Campos, J., Martinez, E., Marrero, K., Silva, Y., Rodriguez, B. L., Suzarte, E., et al. (2003a). Novel type of specialized transduction for CTX or its satellite phage RS1 mediated by filamentous phage VGJ in Vibrio cholerae. J. Bacteriol. 185, 7231-7240. doi: 10.1128/JB.185.24.7231-7240.2003

Campos, J., Martinez, E., Suzarte, E., Rodriguez, B. L., Marrero, K., Silva, Y., et al. (2003b). VGJ, a novel filamentous phage of Vibrio cholerae, integrates into the same chromosomal site as CTX. J. Bacteriol. 185, 5685-5696. doi: 10.1128/JB.185.19.5685-5696.2003

Chun, J., Grim, C. J., Hasan, N. A., Lee, J. H., Choi, S. Y., Haley, B. J., et al. (2009). Comparative genomics reveals mechanism for short-term and longterm clonal transitions in pandemic Vibrio cholerae. Proc. Natl. Acad. Sci. U.S.A. 106, 15442-15447. doi: 10.1073/pnas.0907787106

Das, B., Bischerour, J., and Barre, F. X. (2011a). Molecular mechanism of acquisition of the cholera toxin genes. Indian J. Med. Res. 133, 195-200.

Das, B., Bischerour, J., and Barre, F. X. (2011b). VGJphi integration and excision mechanisms contribute to the genetic diversity of Vibrio cholerae epidemic strains. Proc. Natl. Acad. Sci. U.S.A. 108, 2516-2521. doi: 10.1073/pnas.1017061108

Das, B., Bischerour, J., Val, M. E., and Barre, F. X. (2010). Molecular keys of the tropism of integration of the cholera toxin phage. Proc. Natl. Acad. Sci. U.S.A. 107, 4377-4382. doi: 10.1073/pnas.0910212107

Das, B., Kumari, R., Pant, A., Sen Gupta, S., Saxena, S., Mehta, O., et al. (2014). A novel broad range CTXPhi derived stable integrative expression vector for functional studies. J. Bacteriol. 196, 4071-4080. doi: 10.1128/JB.01966-14

Das, B., Martinez, E., Midonet, C., and Barre, F. X. (2013). Integrative mobile elements exploiting Xer recombination. Trends Microbiol. 21, 23-30. doi: 10.1016/j.tim.2012.10.003

Davies, B. W., Bogard, R. W., Young, T. S., and Mekalanos, J. J. (2012). Coordinated regulation of accessory genetic elements produces cyclic dinucleotides for V. cholerae virulence. Cell 149, 358-370. doi: 10.1016/j.cell.2012. 01.053

Davis, B. M., Kimsey, H. H., Kane, A. V., and Waldor, M. K. (2002). A satellite phage-encoded antirepressor induces repressor aggregation and cholera toxin gene transfer. EMBO J. 21, 4240-4249. doi: 10.1093/emboj/cdf427

Davis, B. M., and Waldor, M. K. (2000). CTXphi contains a hybrid genome derived from tandemly integrated elements. Proc. Natl. Acad. Sci. U.S.A. 97, 8572-8577. doi: 10.1073/pnas.140109997

Demarre, G., Galli, E., and Barre, F. X. (2013). The FtsK family of DNA pumps. Adv. Exp. Med. Biol. 767, 245-262. doi: 10.1007/978-1-4614-5037-5_12

Esposito, D., and Scocca, J. J. (1997). The integrase family of tyrosine recombinases: evolution of a conserved active site domain. Nucleic Acids Res. 25, 3605-3614. doi: 10.1093/nar/25.18.3605

Faruque, S. M., and Mekalanos, J. J. (2003). Pathogenicity islands and phages in Vibrio cholerae evolution. Trends Microbiol. 11, 505-510. doi: 10.1016/j.tim.2003.09.003
Faruque, S. M., and Mekalanos, J. J. (2012). Phage-bacterial interactions in the evolution of toxigenic Vibrio cholerae. Virulence 3, 556-565. doi: 10.4161/viru. 22351

Faruque, S. M., Rahman, M. M., Hasan, A. K., Nair, G. B., Mekalanos, J. J., and Sack, D. A. (2001). Diminished diarrheal response to Vibrio cholerae strains carrying the replicative form of the CTX(Phi) genome instead of CTX(Phi) lysogens in adult rabbits. Infect. Immun. 69, 6084-6090. doi: 10.1128/IAI.69.10.60846090.2001

Frost, L. S., Leplae, R., Summers, A. O., and Toussaint, A. (2005). Mobile genetic elements: the agents of open source evolution. Nat. Rev. Microbiol. 3, 722-732. doi: $10.1038 /$ nrmicrol235

Fullner, K. J., and Mekalanos, J. J. (1999). Genetic characterization of a new type IV-A pilus gene cluster found in both classical and El Tor biotypes of Vibrio cholerae. Infect. Immun. 67, 1393-1404.

Hassan, F., Kamruzzaman, M., Mekalanos, J. J., and Faruque, S. M. (2010). Satellite phage TLCphi enables toxigenic conversion by CTX phage through dif site alteration. Nature 467, 982-985. doi: 10.1038/nature09469

Hazen, T. H., Pan, L., Gu, J. D., and Sobecky, P. A. (2010). The contribution of mobile genetic elements to the evolution and ecology of Vibrios. FEMS Microbiol. Ecol. 74, 485-499. doi: 10.1111/j.1574-6941.2010.00937.x

Heidelberg, J. F., Eisen, J. A., Nelson, W. C., Clayton, R. A., Gwinn, M. L., Dodson, R. J., et al. (2000). DNA sequence of both chromosomes of the cholera pathogen Vibrio cholerae. Nature 406, 477-483. doi: 10.1038/35020000

Heilpern, A. J., and Waldor, M. K. (2000). CTXphi infection of Vibrio cholerae requires the tolQRA gene products. J. Bacteriol. 182, 1739-1747. doi: 10.1128/JB.182.6.1739-1747.2000

Herrington, D. A., Hall, R. H., Losonsky, G., Mekalanos, J. J., Taylor, R. K., and Levine, M. M. (1988). Toxin, toxin-coregulated pili, and the toxR regulon are essential for Vibrio cholerae pathogenesis in humans. J. Exp. Med. 168, 1487-1492. doi: 10.1084/jem.168.4.1487

Huber, K. E., and Waldor, M. K. (2002). Filamentous phage integration requires the host recombinases XerC and XerD. Nature 417, 656-659. doi: 10.1038 /nature00782

Karaolis, D. K., Somara, S., Maneval, D. R. Jr., Johnson, J. A., and Kaper, J. B. (1999). A bacteriophage encoding a pathogenicity island, a type-IV pilus and a phage receptor in cholera bacteria. Nature 399, 375-379. doi: 10.1038/20715

MacDonald, D., Demarre, G., Bouvier, M., Mazel, D., and Gopaul, D. N. (2006). Structural basis for broad DNA-specificity in integron recombination. Nature 440, 1157-1162. doi: 10.1038/nature04643

Mazel, D. (2006). Integrons: agents of bacterial evolution. Nat. Rev. Microbiol. 4 608-620. doi: 10.1038/nrmicro1462

Mazel, D., and Davies, J. (1998). Antibiotic resistance. The big picture. Adv. Exp. Med. Biol. 456, 1-6. doi: 10.1007/978-1-4615-4897-3_1

Mekalanos, J. J. (1985). Cholera toxin: genetic analysis, regulation, and role in pathogenesis. Curr. Top. Microbiol. Immunol. 118, 97-118. doi: 10.1007/978-3642-70586-1_6

Mekalanos, J. J., Swartz, D. J., Pearson, G. D., Harford, N., Groyne, F., and de Wilde, M. (1983). Cholera toxin genes: nucleotide sequence, deletion analysis and vaccine development. Nature 306, 551-557. doi: 10.1038/306551a0

Mercier, R., Petit, M. A., Schbath, S., Robin, S., El Karoui, M., Boccard, F., et al. (2008). The $\mathrm{MatP} / \mathrm{matS}$ site-specific system organizes the terminus region of the E. coli chromosome into a macrodomain. Cell 135, 475-485. doi: 10.1016/j.cell.2008.08.031

Midonet, C., Das, B., Paly, E., and Barre, F. X. (in press). XerD initiates the integration of TLC $\varphi$ into the Vibrio cholerae genome independently of FtsK. Proc. Natl. Acad. Sci. U.S.A. doi: 10.1073/pnas.1404047111

Rajanna, C., Wang, J., Zhang, D., Xu, Z., Ali, A., Hou, Y. M., et al. (2003). The Vibrio pathogenicity island of epidemic Vibrio cholerae forms precise extrachromosomal circular excision products. J. Bacteriol. 185, 6893-6901. doi: 10.1128/JB.185.23.6893-6901.2003

Recchia, G. D., Aroyo, M., Wolf, D., Blakely, G., and Sherratt, D. J. (1999). FtsK-dependent and -independent pathways of Xer site-specific recombination. EMBO J. 18, 5724-5734. doi: 10.1093/emboj/18.20.5724

Rhine, J. A., and Taylor, R. K. (1994). TcpA pilin sequences and colonization requirements for $\mathrm{O} 1$ and $\mathrm{O} 139$ vibrio cholerae. Mol. Microbiol. 13, 1013-1020. doi: 10.1111/j.1365-2958.1994.tb00492.x

Rubin, E. J., Lin, W., Mekalanos, J. J., and Waldor, M. K. (1998). Replication and integration of a Vibrio cholerae cryptic plasmid linked to the CTX prophage. Mol. Microbiol. 28, 1247-1254. doi: 10.1046/j.1365-2958.1998.00889.x 
Sack, D. A., Sack, R. B., Nair, G. B., and Siddique, A. K. (2004). Cholera. Lancet 363, 223-233. doi: 10.1016/S0140-6736(03)15328-7

Sherratt, D. J., and Wigley, D. B. (1998). Conserved themes but novel activities in recombinases and topoisomerases. Cell 93, 149-152. doi: 10.1016/S00928674(00)81566-4

Taylor, R. K., Miller, V. L., Furlong, D. B., and Mekalanos, J. J. (1986). Identification of a pilus colonization factor that is coordinately regulated with cholera toxin. Ann. Sclavo Collana Monogr. 3, 51-61.

Val, M. E., Bouvier, M., Campos, J., Sherratt, D., Cornet, F., Mazel, D., et al. (2005). The single-stranded genome of phage CTX is the form used for integration into the genome of Vibrio cholerae. Mol. Cell 19, 559-566. doi: 10.1016/j.molcel.2005.07.002

Val, M. E., Kennedy, S. P., El Karoui, M., Bonne, L., Chevalier, F., and Barre, F. X. (2008). FtsK-dependent dimer resolution on multiple chromosomes in the pathogen Vibrio cholerae. PLoS Genet. 4:e1000201. doi: 10.1371/journal.pgen. 1000201

Vanden Broeck, D., Horvath, C., and De Wolf, M. J. (2007). Vibrio cholerae: cholera toxin. Int. J. Biochem. Cell Biol. 39, 1771-1775. doi: 10.1016/j.biocel.2007. 07.005

Waldor, M. K., and Mekalanos, J. J. (1996). Lysogenic conversion by a filamentous phage encoding cholera toxin. Science 272, 1910-1914. doi: 10.1126/science.272.5270.1910
Waldor, M. K., Rubin, E. J., Pearson, G. D., Kimsey, H., and Mekalanos, J. J. (1997). Regulation, replication, and integration functions of the Vibrio cholerae CTXphi are encoded by region RS2. Mol. Microbiol. 24, 917-926. doi: 10.1046/j.13652958.1997.3911758.x

Conflict of Interest Statement: The Guest Associate Editor, Jasna Rakonjac, declares that, despite having collaborated on the same research topic as author Bhabatosh Das, the review process was handled objectively and no conflict of interest exists.

Received: 02 August 2014; accepted: 10 November 2014; published online: 28 November 2014.

Citation: Das B (2014) Mechanistic insights into filamentous phage integration in Vibrio cholerae. Front. Microbiol. 5:650. doi: 10.3389/fmicb.2014.00650

This article was submitted to Virology, a section of the journal Frontiers in Microbiology.

Copyright () 2014 Das. This is an open-access article distributed under the terms of the Creative Commons Attribution License (CC BY). The use, distribution or reproduction in other forums is permitted, provided the original author(s) or licensor are credited and that the original publication in this journal is cited, in accordance with accepted academic practice. No use, distribution or reproduction is permitted which does not comply with these terms. 